\title{
Narrative review of anxiety and depression in patients with esophageal cancer: underappreciated and undertreated
}

\author{
Brian Housman, Raja Flores, Dong-Seok Lee \\ Department of Thoracic Surgery, Icahn School of Medicine at Mount Sinai, Mount Sinai Health System, New York, NY, USA \\ Contributions: (I) Conception and design: B Housman; (II) Administrative support: DS Lee, R Flores; (III) Provision of study materials or patients: B \\ Housman; (IV) Collection and assembly of data: B Housman; (V) Data analysis and interpretation: B Housman; (VI) Manuscript writing: All authors; \\ (VII) Final approval of manuscript: All authors. \\ Correspondence to: Raja Flores, MD. Ames Professor of Cardiothoracic Surgery, Chairman, Department of Thoracic Surgery, Mount \\ Sinai Health System, Icahn School of Medicine at Mount Sinai, One Gustave L. Levy Place, Box 1023, New York, NY 10029, USA. \\ Email: raja.flores@mountsinai.org.
}

\begin{abstract}
Depression and anxiety are emotional disorders that commonly affect patients with esophageal cancer. As a result of its high morbidity, mortality, and complication rates, this population is at particularly high risk for developing or exacerbating affective disorders; even when compared to patients with other forms of cancer. Many of the medical conditions and social behaviors that predispose patients to this disease are also independently associated with affective disorders, and likely compound their effects. Unfortunately, in the existing literature, there is wide variability in study design and diagnostic criteria. There is no standard method of evaluation, many studies are limited to written surveys, and widespread mental health screening is not included as a part of routine care. As a result, the prevalence of these illnesses remains elusive. Additionally, psychiatric and psychosocial illness can affect compliance with surveillance and treatment, and gaps in knowledge may ultimately influence patient outcomes and survival. This review will discuss the existing literature on depression and anxiety in patients with esophageal cancer. It will highlight current methods of psychological evaluation, the prevalence of affective disorders in this population, and their effects on treatment, compliance, and outcomes. It will also discuss possible screening tools, treatments and interventions for these comorbid illnesses that may improve oncologic outcomes as well as quality of life.
\end{abstract}

Keywords: Depression; anxiety; affective disorders; Hospital Anxiety and Depression Scale (HADS); esophageal cancer; esophagectomy

Submitted Dec 16, 2020. Accepted for publication Mar 25, 2021.

doi: $10.21037 /$ jtd-20-3529

View this article at: http://dx.doi.org/10.21037/jtd-20-3529

\section{Introduction}

Depression and anxiety are emotional disorders that commonly affect esophageal cancer patients (1). Esophageal cancer is a highly morbid disease with a $10-15 \%$ overall 5 -year survival and a $15-40 \%$ postoperative survival $(2,3)$. At the time of diagnosis, only $25 \%$ of patients are eligible for treatment. When possible, they undergo an extensive course of therapy that can include neoadjuvant chemoradiation followed by surgeries that have a high rate of complications (4-6). As such, this patient population is at particularly high risk for developing or exacerbating affective disorders, even amongst patients with other forms of cancer (7-10). Moreover, psychosocial illness can affect compliance with surveillance and treatment, and ultimately may affect patient outcomes and survival (9-12). This not only affects the individual, but also has the potential to dilute therapeutic data and underestimate the efficacy of modern therapies.

Unfortunately, there is wide variability in both study design and diagnostic criteria that limits our understanding of these disorders and their effects on outcomes. This 
review will discuss the existing literature on depression and anxiety in esophageal cancer patients, their effects on treatment, compliance, outcomes, and possible interventions that may optimize quality of life.

We present the following article in accordance with the Narrative Review reporting checklist (available at http:// dx.doi.org/10.21037/jtd-20-3529).

\section{Methods}

A PubMed search for English-language articles describing affective disorders in the setting of esophageal cancer was conducted from 2000 to 2020. Key words included "anxiety", "depression", "esophageal cancer", "risk factors", "prevalence", "treatment" and all appropriate Boolean operators. We prioritized research from randomized trials that investigated the prevalence and incidence of affective disorders, which involved the use of questionnaires, clinical interview, or formal psychiatric evaluation. Non-randomized studies that discussed risk factors, mechanisms, treatment, and future research were also included from case series, retrospective studies and other review articles. The guidelines, original works and foundational studies from professional societies and leaders in the field were also reviewed. Only articles agreed upon by all authors were included.

\section{Discussion}

\section{The problem with prevalence}

The true prevalence of depression in esophageal cancer patients remains controversial. While clinically relevant psychological distress is commonly accepted to affect about a quarter of cancer patients, reports are highly variable with published ranges anywhere from 5\% to 54\% (13-17). This is likely due to differences in disease-related characteristics, heterogeneity in study design, culture-bound differences in the perceptions of illness, and the limitations of our measurement tools $(7,12-14)$.

The most troubling of these tools is the Hospital Anxiety and Depression Scale (HADS). First introduced by Zigmond in 1983, the HADS is the most commonly utilized evaluation method in the literature (18). It is a 14-question, patient completed self-assessment that grades the likelihood and severity of depression and anxiety on a scale from $0-21$ (19). In the original paper, a patient was asked to fill out a questionnaire, followed by a 20 -minute evaluation by a clinician, and a follow up interview with researchers. Zigmond defined scores of 7 or less as "non-cases", 8-10 as "doubtful cases", and 11 or more as "definite cases".

The HADS is widely regarded as a valid and reliable instrument for the detection of psychological distress in cancer patients (14,20-22). As a result, many studies utilize the HADS as their primary means of evaluation; a widespread decision that has three significant vulnerabilities.

First, the HADS was never intended as a diagnostic tool. In a commonly cited study intended to validate the HADS, Mitchell and colleagues write that for "...the identification of depression, anxiety or distress in cancer settings, the HADS (including subscales) is not recommended as a casefinding instrument but it may... be a suitable addition to a screening programme (sic) (18)." As Mitchell and colleagues caution, the incidence of depression is especially unclear because most studies rely only on screening tools instead of formal diagnostic methods (23). Diagnosis has a greater burden of proof, relying on a formal algorithm, a context of clinical significance, and a minimum duration of time (23). The HADS is deficient in all of these qualities. Nevertheless, since the HADS_and similar screening tools-are being utilized to identify new cases of depression, they serve as de facto diagnostic instruments. Worse, such use is commonly regarded as the gold standard for this type of research (1).

Originally, the HADS score was intended to be a rapid screening method for "clinically significant anxiety and depression in patients attending general medical clinics (19)." It was designed to improve upon the already utilized General Health Questionnaire (GHQ), which was developed by Goldberg in 1972 (24). It is described as "rather long" and only "detects a 'case' [of psychiatric distress] but gives no information about the nature of the psychiatric disorder (19,24)."

While other studies have shown that HADS may be superior to the GHQ, it is difficult to justify the unopposed use of either screening tool to report reliable prevalence data (25). Härter in 2006 published one such study that showed the HADS score to be superior to the GHQ (Depression sensitivity $74 \%$ vs. $52.5 \%$, specificity $80 \%$ vs. $77.9 \%$; Anxiety sensitivity $70.7 \%$ vs. $75.6 \%$, specificity $73 \%$ vs. $55.9 \%$ ). However, since the sensitivity and specificity determined by the authors is qualitatively similar to the study by Mitchell in 2010, these findings also do not support the use of the HADS $(18,25)$.

Second, there is no established standard for a positive threshold. Many of the studies referenced in this review use 
an 8 on the HADS to diagnose anxiety or depression; some using the standard HADS, and some using the subscales. Unfortunately, Zigmond would classify a score of 8 as "doubtful," and unsurprisingly, the use of this value has been shown to yield low sensitivity and specificity $(14,19)$.

Third, even when a higher cut-off is used, the HADS does not perform adequately. In a paper by Härter and colleagues, they publish that the best cutoff points for HADS are 17 for any mental disorder or an anxiety disorder, and 18 for depressive disorders (25). For any mental disorder, this corresponds to a sensitivity of $58.8 \%$, specificity of $77.9 \%$, and positive predictive value of $44 \%$. For a depressive disorder, sensitivity is $73.7 \%$, specificity is $79.5 \%$, and positive predictive value is $30.7 \%$ (25). Even with this higher threshold, this data suggests substantial limitations in the HADS to establish accurate diagnoses.

\section{Are we using the right definitions?}

Complicating matters further, traditional psychiatric definitions of axis I disease may not even be valid in the cancer patient population. Keller and colleagues performed a study in 2004 that compared the HADS to physician and nursing evaluation, and structured clinical interview in cancer patients (14). A total of 186 patients were evaluated by HADS, 181 were rated by medical staff, and 78 consented to formal psychiatric assessment. All 186 patients filled out the HADS questionnaire. Using a threshold of $\geq 11,13.9 \%$ were found to have depression and $19.1 \%$ were found to have anxiety. Interestingly, a value of $\geq 16$ was defined by the authors to be closest to DSM-IV diagnostic criteria. When this standard was applied, 19 of the 22 patients with a current psychiatric disorder were identified (sensitivity $86 \%$ ), and 48 out of 55 patients without a psychiatric illness were accurately identified as non-cases (specificity $87 \%$ ). Of those, 181 patients were evaluated by medical staff using a questionnaire created by the authors. Surgeons and nurses taking care of these patients interpreted that $52.7 \%$ and $56.9 \%$ respectively had symptoms suggestive of a formal DSM-IV diagnosis and 55\% and $54.5 \%$ were distressed (14). While the correlations between these findings were low-Kappa values range from 0.15 to 0.23 - it does raise an important point. If clinicians believe that more patients are suffering emotional distress than those with diagnosable disease, the need for psychosocial support may be broader than we predict. Depression is only one disease in a spectrum of affective disorders, and traditional criteria may not even apply to this patient population. As Mitchell describes, the DSM is "...generic and might not be appropriate in cancer settings (23).”

\section{The virtue of questionnaires}

Despite these limitations, the HADS has substantial clinical utility. Whether due to heavy workloads or lack of formal training, oncologic doctors and nurses have been shown to be poor identifiers of psychological distress $(11,14,23)$. In a study performed by Newell in 1998, oncologists' specificity in detecting clinical anxiety and depression was $17 \%$ and $6 \%$ (11). However, those same oncologists were able to identify much higher levels of need than patients were initially able to report. This resulted in sensitivity rates of major physical symptoms up to $80 \%$ (11). These findings suggest that while oncologists' knowledge of and relationship with patients resulted in an awareness of physical problems, they were unable to identify psychological distress. In meta-analysis of multiple studies, Lampic and colleagues reported that the correlation between patient and staff perceptions of depression and anxiety ranged from 0.21 to 0.35 (26). However, significant variability amongst studies made interpretation of these findings difficult. Lampic reports that correlations of $<0.35$ were regarded by some authors as being able to interpret mood states with "some accuracy," while others interpreted values from 0.31 to 0.50 as clinicians being unable to "accurately determine what the patients felt (26-28)." Unless all patients undergo formal psychiatric evaluation, some form of diagnostic adjunct is necessary to assist with the limitations of untrained clinicians.

The HADS is not the only questionnaire-based evaluation of mental health. The Brief Symptom Inventory (BSI), the Symptom Checklist 90 (SCL-90), the Minnesota Multiphasic Personality Inventory (MMPI), the Beck Depression Inventory (BDI), and the Hamilton Rating Scale for Depression (Ham-D) have all been utilized with comparable utility to the HADS (7). While the prevalence of depression and anxiety vary depending on the screening instrument used, the HADS is most frequently utilized in the literature (29). A full evaluation of each of these methods is beyond the scope of this review.

Unfortunately, there is a paucity of studies that do not rely on a patient-based questionnaire. Singer, noting similar concerns, performed a meta-analysis of studies using a structured clinical interview. With appropriate exclusion criteria and no restrictions on date of publication, Singer 
and colleagues found only 8 studies. While none of the papers included esophageal cancer patients, the published rates of depression ranged from $23 \%$ among breast cancer patients in Turkey to $53 \%$ among elderly patients with unspecified tumor in Uganda (1).

$\mathrm{Hu}$ and colleagues in 2014, noting that most studies involve small populations, performed a retrospective analysis of 28,454 patients; 14,227 with esophageal cancer and 14,227 matched for age, sex, common-comorbidity and enrollment date (8). Diagnosis was established by searching discharge diagnosis codes and new prescriptions for anxiety or depression medication, criteria that require formal evaluation by a psychiatrist. They found that of the 28,454 patients, $990(3.5 \%)$ were diagnosed with anxiety and depression disorders: 382 in the esophageal cancer group with a cumulative incidence of 20.1 per 1,000 patientyears, and 608 in the matched cohort 9.2 per 1,000 patientyears (8). While this study is important, we were unable to find another study with reported incidence in the form of patient-years, making comparison difficult.

In 2011, Mitchell performed a meta-analysis on the prevalence of affective disorders on patients in the oncological, hematological and palliative care setting (23). He was able to identify 94 studies (24 in the palliative setting and 70 in the non-palliative setting) for a total pool of 14,078 patients; all of whom were evaluated by clinical interview. He found $16.3 \%$ had major depression or a major depressive episode, $14.9 \%$ had major depression only, $19.2 \%$ had minor depression only, $20.7 \%$ had any depression, $31.6 \%$ had depression or adjustment disorder, and $38.2 \%$ had depression or adjustment disorder or an anxiety disorder (23). While this analysis includes subjects with many forms of disease, these findings still serve as an important touchstone.

Previously cited, the study by Keller and colleagues identified 22 of the 78 (28\%) patients who underwent formal psychiatric evaluation of a total group of 186 (14). But this study was not performed exclusively on esophageal cancer patients, and was not designed to investigate incidence.

\section{Timing}

Adding to the heterogeneity in the literature, studies perform patient evaluations at different times during treatment. Unfortunately, prevalence is known to change over time and increase during critical events: initial diagnosis, the beginning of treatment, evaluation of response to treatment, after recurrence, and during terminal stages (29-31). Patients appear to experience the greatest psychological distress and impairments in quality of life during the first year following diagnosis, but feelings of anxiety and depression may remain elevated for up to 4 years (32-34). Of particular note, Fang and colleagues found that cancer patients are the most vulnerable for the first 3 months after diagnosis, reporting a nearly 16-fold increased risk of suicide compared with cancer-free patients (35,36).

Hellstadius and colleagues investigated the incidence of affective disorders at three stages in esophageal cancer patients; before surgery, 6 months post-surgery, and 12 months postsurgery. Anxiety was present in 33\%, 28\% and $37 \%$ while depression was present in 20\%, $27 \%$, and $32 \%$ respectively (9). These findings call into question the timing for psychological evaluation and whether studies relying on single time points accurately reflect emotional distress.

\section{Risk factors}

While the actual prevalence of affective disorders may be unknown, esophageal cancer patients represent an especially high-risk population. Multiple studies have reported that depression and anxiety are significantly higher in esophageal cancer than both the general population and among other forms of cancer (7-10).

In a cohort of 28,454 matched hospitalized patients, $\mathrm{Hu}$ and colleagues found that having esophageal cancer increased the risk of new depression by a hazard ratio of 2.24 (8).

Hong in 2014 investigated the prevalence of anxiety and depression in 1,217 Chinese cancer patients using a HADS threshold $>11$. Compared to a rate of $3.8 \%$ in the general population, anxiety and depression among all cancer patient were identified in $6.49 \%$ and $66.72 \%$ respectively. Among esophageal cancer patients, $75.81 \%$ were found to have depression; higher than patients with gastric cancer $(63.40 \%)$ and the second most frequent overall after lung cancer $(77.19 \%)(13)$.

There is a large body of work in the literature that has investigated the causes for this observation. Limitations in activity status and reduced quality of life are often central to most risk factors $(34,37,38)$. Unfortunately, the specific causes are presented haphazardly; often described in qualitatively different ways, through quantitatively different variables, and rarely with the standardized weight of hazard 
ratios. There appear to be six major categories of risk factors, which are described below.

\section{The diagnosis is traumatic}

At the time of diagnosis, most patients will not be surgical candidates. Esophageal cancer carries a $10-15 \%$ overall 5 -year survival, and a $15-40 \%$ postoperative survival $(2,3,39)$. Patients are often diagnosed at a late stage, when only $25 \%$ will be eligible for treatment; which places it among the least favorable of all cancers (4-6,8). Due to its poor outcomes, new diagnosis with esophageal cancer has been described as a "death sentence," and is among the highest risks for causing depression and anxiety (8,34,40-42). It has even been suggested that the experience may evoke symptoms of post-traumatic stress $(8,40)$. As described earlier, the first months to year following diagnosis are the highest risk for depression and suicide, with an up to 16-fold increase compared to the general population $(29-31,35,36)$.

\section{Postoperative complications are common and significantly affect quality of life}

Following diagnosis, and when possible, surgery for esophageal cancer is invasive and comprehensive. Treatment often requires large incisions, gastrointestinal reconstruction, and enteral feeding access, and may require neoadjuvant chemoradiation (4,9,43-46). Collectively, the different types of esophagectomy remain some of the highest-risk elective operations, with peri-operative mortality reported from $3 \%$ to $20 \%(45,46)$. Starting treatment has been shown to decrease rates of anxiety and depression following diagnosis, unless treatment has palliative intent (33).

Rates of in-hospital complications (including wound infection, pneumonia, pneumothorax, anastomotic leak, and mediastinitis) can be as high as $40-50 \%$, and are known risk factors for depression $(9,47)$. The resulting physical symptoms experienced by patients-pain, inadequate symptom treatment, prolonged hospital stay and limitations in activity status-are also significant risk factors, and may explain this phenomenon better than the complication itself $(13,14,23,37,38)$.

Dysphagia, one of the most common symptoms before diagnosis, is another significant cause of emotional distress $(9,48)$. Unfortunately, even following surgery, more than a third of patients will report persistent dysphagia for up to 5 years (49). In addition to affecting overall quality of life, re-experiencing symptoms that were present before treatment compound the emotional distress of the morbidity alone (9).

Long term complications, including strictures, post- vagotomy syndromes, dumping syndrome, weight changes, limitations in eating habits, and the ever-present risk of recurrence, are also known causes of emotional distress $(7,8,42)$.

\section{Social consequences and returning to normalcy}

Even with an uncomplicated therapeutic course, some patients may experience difficulty returning to their normal lives. Many patients describe some form of dysphagia or food intolerance following surgery (49). This results in dietary changes, weight loss, and a recovery process that feels similar to symptoms experienced before treatment (42). Biofeedback mechanisms of satiety, nausea and vomiting often have to be re-learned by patients, which can significantly affect gatherings and day-to-day interaction (42). Enteral Feeding is a routine element of esophagectomy and is also known to be an independent risk factor for developing depression for similar reasons $(8,50)$. The regular use of tube feeds requires bulky equipment, professional care, and formula-based diet. As a result, patients may feel socially isolated, which not only depresses mood, but may reduce support mechanisms, and increase the result of depression and suicidal thoughts $(7,8,23,42)$.

Similarly, patients living alone were more likely to report anxiety than patients who are married or cohabitating. This is likely due to the stress-buffering effect of social support, which has been associated with both lower anxiety and depression in cancer patients $(9,51)$.

\section{Biochemical theories}

There is some evidence to suggest that there may be biochemical mechanisms that predispose patients to depression. Cerebrovascular disease, cirrhosis, chronic inflammatory changes, impaired serotonergic neurotransmission, current and former smoking have all been shown to increase the risk of depression in cancer patients $(8,38,52-54)$.

Substance abuse is also higher among survivors of esophageal cancer than both the general population and other forms of cancer (55) In a study from South Korea, $19.4 \%$ of patients with psychological distress exhibited some form of substance abuse; $90 \%$ of which was alcohol related (55). Since alcohol is a known risk factor for esophageal squamous cell carcinoma, this likely reflects a combination of both pre-existing users and post-diagnosis coping mechanisms (56). Additionally, since cirrhosis also increases the risk of depression, multiple abuse-related factors appear to influence depression in this already highrisk population $(52,55)$. Causative or correlative effects of 
substance abuse need to be further explored to determine how best to treat and/or prevent this comorbidity in esophageal cancer survivors.

The presence of metastases has also been associated with anxiety in patients with gastrointestinal cancers, but not breast cancer (7). This suggests that factors specific to cancer biology may play a role instead of the psychology effect of the metastases themselves. (7). For example, a study by Cheng and colleagues found that the mitogen-activated protein kinase molecule, p38, was associated with reduced survival, higher rates of recurrence, lymph node metastases and also depression (57).

On the other hand, Hellstadius and colleagues, in their paper investigating rates of affective disorders over time showed that changes in anxiety were unrelated to tumor recurrence and subsequent treatment. Though they were not able to prove the same about changes in depression, it does suggest that there was no relationship to tumor-related biochemical factors (9).

Though controversial and vulnerable to confounding, these studies reveal that our understanding of cancer-related depression requires further investigation.

\section{Psychiatric factors}

Typical research into affective disorders focuses on new cases after diagnosis with esophageal cancer. However, it must be noted that patients with pre-existing anxiety or depression have a higher likelihood of developing the other $(38,58)$. Moreover, patients suffering from both will experience more severe symptoms and less improvement after treatment (7).

\section{Demographic factors}

Age, gender, and even education have all been suggested as risk factors for anxiety or depression, but are inconsistent in the literature $(8,9,13)$. Of note, multiple studies have suggested female gender is an independent risk factor for anxiety but not depression $(9,14,38,59)$. To the authors' knowledge, no study was able to identify a mechanism for this finding, which may simply reflect an increased willingness to communicate.

\section{Effects on outcomes}

In addition to decreasing overall quality of life, depression is known to exert physical consequences on cancer patients. While the mechanisms remain elusive, multiple studies report worse postoperative outcomes, decreased compliance, and increased rates of mortality $(7,10,12,60-63)$.

A study by Wikman and colleagues showed that among 1,615 patients who underwent surgery for esophageal cancer, the 2-year cumulative incidence for inpatient and outpatient psychiatric treatment was $2.5 \%$ and $4.2 \%$, respectively (10). New cases of psychiatric disease were associated with an increased risk of mortality, with a hazard ratio of 1.65 for inpatient psychiatric care, 1.93 for outpatient care, and 2.77 for patients treated with psychotropic medication. Since the cause of death was recurrence in greater than $80 \%$ of cases, this also supports the possibility of a biochemical etiology (10).

Two meta-analyses from Pinquart and Satin also showed that depressed patients had an increased risk of mortality from $5 \%$ to $40 \%$ compared to matched subjects. In both studies, this relationship remained when subjects were controlled for clinical and medical factors $(61,62)$.

Dimatteo and colleagues published a meta-analysis of 13 studies that found depressed patients were three times as likely to be non-compliant, and found that $63.5 \%$ of all non-compliant patients to be depressed (12). They write that reasons for non-compliance include a disbelief in the efficacy of treatment, the presence of barriers including adverse effects and financial limitations, and a lack of help and support from family members (12).

Spiegel in 2003 described three possible mechanisms for this phenomenon:

(I) Immunologic and Neuroendocrine dysfunction including disruption of the HypothalamicPituitary-Adrenal Axis, and Adrenocortical Function;

(II) Depressed patients are less likely to follow-up with screening, disease treatment or general health recommendations;

(III) Many symptoms of depression are similar to those of cancer and the side-effects of chemotherapy or surgery (e.g., sleep disturbances, decreased appetite, fatigue, difficulty concentrating) (64).

Depressed patients may also be less interested in cultivating relationships with healthcare providers, have a limited capacity to understand their disease process, are less likely to seek social support, and may experience less benefit from it when they do $(12,61)$. As described previously, depression also confers a known risk for suicide, and likely other non-cancer causes of death $(8,35,65)$.

\section{Interventions}

To the authors' knowledge, there are no studies that 
establish criteria for treatment of cancer patients with affective disorders. This is likely due to limitations in our ability to identify these disorders, and the widespread belief that "normal" depression and anxiety behave the same as their cancer-related presentations.

Fortunately, there are several studies that are investigating possible interventions for cancer patients with depression. The use of psychotherapy is controversial in the literature. A meta-analysis by Akechi and colleagues in 2008 found that for advanced cancers, psychotherapy was effective in treating depression (66). However, another meta-analysis performed in 2017 authored by three of the same authors was less optimistic, describing that "[low] quality evidence suggests that psychotherapy is moderately more effective for the amelioration of symptoms of depression among advanced, incurable cancer patients... (67)." Heterogeneity among the studies was noted as another barrier in drawing proper conclusions.

Lydiatt and colleagues performed a randomized placebocontrolled study into the use of citalopram prophylaxis in non-depressed patients with head and neck cancer (68). When initiated before radiation, $13.8 \%$ of patients taking citalopram developed depression versus $38.7 \%$ in the placebo group (68). When started before undergoing surgery, $6.5 \%$ of patients taking escitalopram developed depression versus $11.8 \%$ in the placebo group (68).

Cognitive behavioral therapy (CBT) has also been shown to be an effective treatment for the treatment of functional gastrointestinal conditions, including irritable bowel syndrome, abdominal pain, and constipation $(69,70)$. While these conditions share few similarities with esophageal cancer, many side effects of esophagogastric resection and bilateral vagotomy will have similar presentations. The utility of CBT and biofeedback training may benefit these patients and is worth further investigation.

Swallowing training for dysphagia has been shown to reduce the severity of depression in patients with tongue cancer (71). Though the pathophysiology of dysphagia will be different for esophageal patients, it remains one of the most common and most significant sources of emotional distress, and may warrant investigation.

Hirayama and colleagues have investigated the use of behavioral activation therapy (BAT); a form of treatment that promotes participation in daily activities instead of cognitive or pharmacologic intervention. Patients are commonly hesitant to confront death, symptom progression, and eventual loss of control. BAT seeks to alleviate depression symptoms by focusing on involvement in activities that patients enjoy, especially since participation is usually avoided (72).

As described by Hirayama ... "(I) individuals suffering from depressive or anxiety conditions experience avoidance and decreased participation in normal activities; (II) this leads to decreased opportunities for them to experience joy; (III) they feel that they are overwhelmed by hardships; (IV) the value they place on their lives and themselves is diminished; (V) they pay more attention to negative information; (VI) they eventually experience more distress and depression; (VII) BAT facilitates activities they value and breaks this vicious cycle; and (VIII) this improves the depressive condition and (IX) leads to improvement in quality of life (72)."

While this study involved only ten patients, and none of the patients had esophageal cancer, the efficacy of BAT in treating depression is well-established and may be particularly suited for patients with cancer (72-74).

\section{Summary}

Depression and anxiety are common, and likely underappreciated, in esophageal cancer. The high morbidity, mortality, and rate of complications make this disease particularly high risk for emotional distress and psychiatric illness. It is possible that a fundamental overhaul of the definition of affective disorders-as they relate to cancer patients-may also be necessary.

It is also arguable that formal psychological evaluation may need to be included as a standard element of preoperative work-up and continued during the postoperative course.

This is not unprecedented. In 1991, during the early days of bariatric surgery, the national institutes of health released a consensus statement that patients must be "... selected carefully after evaluation by a multi-disciplinary team with medical, surgical, psychiatric, and nutritional expertise (75)." This was established because of the risks of bariatric procedures, and the need for long-term behavioral change following what were considered elective operations (76). Surgery for esophageal cancer has a comparable and often more severe risk profile, and causes similarly restrictive and malabsorptive changes to gastrointestinal physiology. Cancer patients, who endure arguably greater physical and emotional demands, should be worthy of similar considerations. Adding psychiatric evaluation to a standard perioperative evaluation would serve both to prospectively identify actual prevalence as well as provide treatment to 
affected patients. Devoted "oncologic-psychiatry" and peer support should be offered to every patient with a new cancer diagnosis; both to improve quality of life and ensure maximal compliance to treatment.

If not just life, but quality of life, is the goal of esophageal cancer treatment, then mental well-being must be emphasized as much as surgery.

\section{Acknowledgments}

Funding: None.

\section{Footnote}

Reporting Checklist: The authors have completed the Narrative Review reporting checklist. Available at http:// dx.doi.org/10.21037/jtd-20-3529

Conflicts of Interest: All authors have completed the ICMJE uniform disclosure form (available at http://dx.doi. org/10.21037/jtd-20-3529). RMF serves as an unpaid editorial board member of Fournal of Thoracic Disease from Sep 2020 to Aug 2022. The other authors have no conflicts of interest to declare.

Ethical Statement: The authors are accountable for all aspects of the work in ensuring that questions related to the accuracy or integrity of any part of the work are appropriately investigated and resolved.

Open Access Statement: This is an Open Access article distributed in accordance with the Creative Commons Attribution-NonCommercial-NoDerivs 4.0 International License (CC BY-NC-ND 4.0), which permits the noncommercial replication and distribution of the article with the strict proviso that no changes or edits are made and the original work is properly cited (including links to both the formal publication through the relevant DOI and the license). See: https://creativecommons.org/licenses/by-nc-nd/4.0/.

\section{References}

1. Singer S, Das-Munshi J, Brähler E. Prevalence of mental health conditions in cancer patients in acute care--a metaanalysis. Ann Oncol 2010;21:925-30.

2. Huang FL, Yu SJ. Esophageal cancer: Risk factors, genetic association, and treatment. Asian J Surg 2018;41:210-5.

3. Lagergren J, Mattsson F, Mattson F. Diverging trends in recent population-based survival rates in oesophageal and gastric cancer. PLoS One 2012;7:e41352.

4. Shapiro J, van Lanschot JJB, Hulshof MCCM, et al. Neoadjuvant chemoradiotherapy plus surgery versus surgery alone for oesophageal or junctional cancer (CROSS): long-term results of a randomised controlled trial. Lancet Oncol 2015;16:1090-8.

5. Thompson SK, Ruszkiewicz AR, Jamieson GG, et al. Improving the accuracy of TNM staging in esophageal cancer: a pathological review of resected specimens. Ann Surg Oncol 2008;15:3447-58.

6. Rutegård M, Charonis K, Lu Y, et al. Populationbased esophageal cancer survival after resection without neoadjuvant therapy: an update. Surgery 2012;152:903-10.

7. Brintzenhofe-Szoc KM, Levin TT, Li Y, et al. Mixed anxiety/depression symptoms in a large cancer cohort: prevalence by cancer type. Psychosomatics 2009;50:383-91.

8. Hu LY, Ku FC, Wang YP, et al. Anxiety and depressive disorders among patients with esophageal cancer in Taiwan: a nationwide population-based study. Support Care Cancer 2015;23:733-40.

9. Hellstadius Y, Lagergren J, Zylstra J, et al. A longitudinal assessment of psychological distress after oesophageal cancer surgery. Acta Oncol 2017;56:746-52.

10. Wikman A, Ljung R, Johar A, et al. Psychiatric morbidity and survival after surgery for esophageal cancer: a population-based cohort study. J Clin Oncol 2015;33:448-54.

11. Newell S, Sanson-Fisher RW, Girgis A, et al. How well do medical oncologists' perceptions reflect their patients' reported physical and psychosocial problems? Data from a survey of five oncologists. Cancer 1998;83:1640-51.

12. DiMatteo MR, Lepper HS, Croghan TW. Depression is a risk factor for noncompliance with medical treatment: meta-analysis of the effects of anxiety and depression on patient adherence. Arch Intern Med 2000;160:2101-7.

13. Hong JS, Tian J. Prevalence of anxiety and depression and their risk factors in Chinese cancer patients. Support Care Cancer 2014;22:453-9.

14. Keller M, Sommerfeldt S, Fischer C, et al. Recognition of distress and psychiatric morbidity in cancer patients: a multi-method approach. Ann Oncol 2004;15:1243-9.

15. Massie MJ. Prevalence of depression in patients with cancer. J Natl Cancer Inst Monogr 2004;(32):57-71.

16. Ashraff S, Gupta AK, Chaudhury S, et al. Effect of short-term Psychiatric Intervention in Cancer Patients. Med J Armed Forces India 2004;60:109-12.

17. So WK, Marsh G, Ling WM, et al. Anxiety, depression 
and quality of life among Chinese breast cancer patients during adjuvant therapy. Eur J Oncol Nurs 2010;14:17-22.

18. Mitchell AJ, Meader N, Symonds P. Diagnostic validity of the Hospital Anxiety and Depression Scale (HADS) in cancer and palliative settings: a meta-analysis. J Affect Disord 2010;126:335-48.

19. Zigmond AS, Snaith RP. The hospital anxiety and depression scale. Acta Psychiatr Scand 1983;67:361-70.

20. Hopwood P, Howell A, Maguire P. Screening for psychiatric morbidity in patients with advanced breast cancer: validation of two self-report questionnaires. Br J Cancer 1991;64:353-6.

21. Ibbotson T, Maguire P, Selby P, et al. Screening for anxiety and depression in cancer patients: the effects of disease and treatment. Eur J Cancer 1994;30A:37-40.

22. Aass N, Fosså SD, Dahl AA, et al. Prevalence of anxiety and depression in cancer patients seen at the Norwegian Radium Hospital. Eur J Cancer 1997;33:1597-604.

23. Mitchell AJ, Chan M, Bhatti H, et al. Prevalence of depression, anxiety, and adjustment disorder in oncological, haematological, and palliative-care settings: a meta-analysis of 94 interview-based studies. Lancet Oncol 2011;12:160-74.

24. Goldberg DP. The detection of psychiatric illness by questionnaire; a technique for the identification and assessment of non-psychotic psychiatric illness. London, New York: Oxford Univ. Press; 1972.

25. Härter M, Woll S, Wunsch A, et al. Screening for mental disorders in cancer, cardiovascular and musculoskeletal diseases. Comparison of HADS and GHQ-12. Soc Psychiatry Psychiatr Epidemiol 2006;41:56-62.

26. Lampic C, Sjödén PO. Patient and staff perceptions of cancer patients' psychological concerns and needs. Acta Oncol 2000;39:9-22.

27. Slevin ML, Plant H, Lynch D, et al. Who should measure quality of life, the doctor or the patient? Br J Cancer 1988;57:109-12.

28. Sensky T, Dennehy M, Gilbert A, et al. Physicians' perceptions of anxiety and depression among their outpatients: relationships with patients and doctors' satisfaction with their interviews. J R Coll Physicians Lond 1989;23:33-8.

29. Linden W, Vodermaier A, Mackenzie R, et al. Anxiety and depression after cancer diagnosis: prevalence rates by cancer type, gender, and age. J Affect Disord 2012;141:343-51.

30. Watson M. Is 'bedside medicine' returning to oncology?
Acta Oncol 2000;39:1-3.

31. Hopwood P, Sumo G, Mills J, et al. The course of anxiety and depression over 5 years of follow-up and risk factors in women with early breast cancer: results from the UK Standardisation of Radiotherapy Trials (START). Breast 2010;19:84-91.

32. Dempster M, McCorry NK, Brennan E, et al. Psychological distress among survivors of esophageal cancer: the role of illness cognitions and coping. Dis Esophagus 2012;25:222-7.

33. Bergquist H, Ruth M, Hammerlid E. Psychiatric morbidity among patients with cancer of the esophagus or the gastro-esophageal junction: a prospective, longitudinal evaluation. Dis Esophagus 2007;20:523-9.

34. Visser MR, van Lanschot JJ, van der Velden J, et al. Quality of life in newly diagnosed cancer patients waiting for surgery is seriously impaired. J Surg Oncol 2006;93:571-7.

35. Fang F, Fall K, Mittleman MA, et al. Suicide and cardiovascular death after a cancer diagnosis. N Engl J Med 2012;366:1310-8.

36. Björkenstam C, Edberg A, Ayoubi S, et al. Are cancer patients at higher suicide risk than the general population? Scand J Public Health 2005;33:208-14.

37. Hulbert-Williams N, Neal R, Morrison V, et al. Anxiety, depression and quality of life after cancer diagnosis: what psychosocial variables best predict how patients adjust? Psychooncology 2012;21:857-67.

38. Boyes AW, Girgis A, D'Este CA, et al. Prevalence and predictors of the short-term trajectory of anxiety and depression in the first year after a cancer diagnosis: a population-based longitudinal study. J Clin Oncol 2013;31:2724-9.

39. Liu SZ, Wang B, Zhang F, et al. Incidence, survival and prevalence of esophageal and gastric cancer in Linzhou city from 2003 to 2009. Asian Pac J Cancer Prev 2013;14:6031-4.

40. Amir M, Ramati A. Post-traumatic symptoms, emotional distress and quality of life in long-term survivors of breast cancer: a preliminary research. J Anxiety Disord 2002;16:195-206.

41. Tytgat GN, Bartelink H, Bernards R, et al. Cancer of the esophagus and gastric cardia: recent advances. Dis Esophagus 2004;17:10-26.

42. McCorry NK, Dempster M, Clarke C, et al. Adjusting to life after esophagectomy: the experience of survivors and carers. Qual Health Res 2009;19:1485-94.

43. Reed C. Technique of Open Ivor Lewis Esophagectomy. 
Operative Techniques in Thoracic and Cardiovascular Surgery 2009;14:160-75.

44. Schieman C, Wigle DA, Deschamps C, et al. Patterns of operative mortality following esophagectomy. Dis Esophagus 2012;25:645-51.

45. Markar S, Gronnier C, Duhamel A, et al. Pattern of Postoperative Mortality After Esophageal Cancer Resection According to Center Volume: Results from a Large European Multicenter Study. Ann Surg Oncol 2015;22:2615-23.

46. Raymond DP, Seder CW, Wright CD, et al. Predictors of Major Morbidity or Mortality After Resection for Esophageal Cancer: A Society of Thoracic Surgeons General Thoracic Surgery Database Risk Adjustment Model. Ann Thorac Surg 2016;102:207-14.

47. Viklund P, Lindblad M, Lu M, et al. Risk factors for complications after esophageal cancer resection: a prospective population-based study in Sweden. Ann Surg 2006;243:204-11.

48. Lagergren J, Lagergren P. Oesophageal cancer. BMJ 2010;341:c6280.

49. Derogar M, Lagergren P. Health-related quality of life among 5-year survivors of esophageal cancer surgery: a prospective population-based study. J Clin Oncol 2012;30:413-8.

50. Chen AM, Daly ME, Vazquez E, et al. Depression among long-term survivors of head and neck cancer treated with radiation therapy. JAMA Otolaryngol Head Neck Surg 2013;139:885-9.

51. Parker PA, Baile WF, de Moor C, et al. Psychosocial and demographic predictors of quality of life in a large sample of cancer patients. Psychooncology 2003;12:183-93.

52. Bianchi G, Marchesini G, Nicolino F, et al. Psychological status and depression in patients with liver cirrhosis. Dig Liver Dis 2005;37:593-600.

53. Nardelli S, Pentassuglio I, Pasquale C, et al. Depression, anxiety and alexithymia symptoms are major determinants of health related quality of life (HRQoL) in cirrhotic patients. Metab Brain Dis 2013;28:239-43.

54. Shen Q, Huang Z, Zhang L. Anxiety and depression in patients with liver cirrhosis. Radiology 2013;269:622-3.

55. Heo J, Noh OK. Psychiatric comorbidities among patients with esophageal cancer in South Korea: a nationwide population-based, longitudinal study. J Thorac Dis 2020;12:1312-9.

56. Islami F, Fedirko V, et al. Alcohol drinking and esophageal squamous cell carcinoma with focus on light-drinkers and never-smokers: a systematic review and meta-analysis. Int
J Cancer 2011;129:2473-84.

57. Cheng Y, Qiao Z, Dang C, et al. p38 predicts depression and poor outcome in esophageal cancer. Oncol Lett 2017;14:7241-9.

58. Hirschfeld RM. The Comorbidity of Major Depression and Anxiety Disorders: Recognition and Management in Primary Care. Prim Care Companion J Clin Psychiatry 2001;3:244-54.

59. Hellstadius Y, Lagergren J, Zylstra J, et al. Prevalence and predictors of anxiety and depression among esophageal cancer patients prior to surgery. Dis Esophagus 2016;29:1128-34.

60. Greer JA, Pirl WF, Park ER, et al. Behavioral and psychological predictors of chemotherapy adherence in patients with advanced non-small cell lung cancer. J Psychosom Res 2008;65:549-52.

61. Pinquart M, Duberstein PR. Depression and cancer mortality: a meta-analysis. Psychol Med 2010;40:1797-810.

62. Satin JR, Linden W, Phillips MJ. Depression as a predictor of disease progression and mortality in cancer patients: a meta-analysis. Cancer 2009;115:5349-61.

63. Hamer M, Chida Y, Molloy GJ. Psychological distress and cancer mortality. J Psychosom Res 2009;66:255-8.

64. Spiegel D, Giese-Davis J. Depression and cancer: mechanisms and disease progression. Biol Psychiatry 2003;54:269-82.

65. Akechi T, Okuyama T, Sugawara Y, et al. Suicidality in terminally ill Japanese patients with cancer. Cancer 2004;100:183-91.

66. Akechi T, Okuyama T, Onishi J, et al. Psychotherapy for depression among incurable cancer patients. Cochrane Database Syst Rev 2008;(2):CD005537.

67. Okuyama T, Akechi T, Mackenzie L, et al. Psychotherapy for depression among advanced, incurable cancer patients: A systematic review and meta-analysis. Cancer Treat Rev 2017;56:16-27.

68. Lydiatt WM, Denman D, McNeilly DP, et al. A randomized, placebo-controlled trial of citalopram for the prevention of major depression during treatment for head and neck cancer. Arch Otolaryngol Head Neck Surg 2008;134:528-35.

69. Palsson OS, Whitehead WE. Psychological treatments in functional gastrointestinal disorders: a primer for the gastroenterologist. Clin Gastroenterol Hepatol 2013;11:208-216; quiz e222-203.

70. Riehl ME, Kinsinger S, Kahrilas PJ, et al. Role of a health psychologist in the management of functional esophageal complaints. Dis Esophagus 2015;28:428-36. 
71. Zhang L, Huang Z, Wu H, et al. Effect of swallowing training on dysphagia and depression in postoperative tongue cancer patients. Eur J Oncol Nurs 2014;18:626-9.

72. Hirayama T, Ogawa Y, Yanai Y, et al. Behavioral activation therapy for depression and anxiety in cancer patients: a case series study. Biopsychosoc Med 2019;13:9.

73. Ekers D, Webster L, Van Straten A, et al. Behavioural activation for depression; an update of meta-analysis of effectiveness and sub group analysis. PLoS One 2014;9:e100100.

Cite this article as: Housman B, Flores R, Lee DS. Narrative review of anxiety and depression in patients with esophageal cancer: underappreciated and undertreated. J Thorac Dis 2021;13(5):3160-3170. doi: 10.21037/jtd-20-3529
74. Richards DA, Rhodes S, Ekers D, et al. Cost and Outcome of BehaviouRal Activation (COBRA): a randomised controlled trial of behavioural activation versus cognitivebehavioural therapy for depression. Health Technol Assess 2017;21:1-366.

75. Hubbard VS, Hall WH. Gastrointestinal Surgery for Severe Obesity. Obes Surg 1991;1:257-65.

76. Snyder AG. Psychological assessment of the patient undergoing bariatric surgery. Ochsner J 2009;9:144-8. 